(whereby patients were followed up and given ADT on detection of clinical recurrence, other than prostate-specific-antigen recurrence; $n=51$ ). Baseline patient characteristics were similar between groups.

Over a median follow-up of 11.9 years, 17 patients $(36 \%)$ receiving immediate ADT and 28 patients (55\%) receiving deferred ADT died. Significantly better overall, progression-free and prostate-cancer-specific survival were observed in patients receiving immediate ADT $(P=0.04$, $P<0.0001$ and $P=0.0004$, respectively). At the end of follow-up, 22 patients (47\%) allocated immediate ADT were alive without detectable disease, compared with 7 patients (14\%) who had been allocated deferred ADT. ADT was generally well tolerated.

Compared with deferring therapy until there was clinical evidence of recurrence, immediate ADT seemed to improve survival in men with node-positive clinically localized prostate cancer who had undergone radical prostatectomy and lymphadenectomy. Further studies are needed, however, to determine whether early ADT is beneficial in less-aggressive prostate cancers.

Original article Messing EM et al. (2006) Immediate versus deferred androgen deprivation treatment in patients with node-positive prostate cancer after radical prostatectomy and pelvic lymphadenectomy. Lancet Oncol 7: 472-479

\section{IMP3: a new prognostic biomarker for renal-cell carcinoma?}

Preliminary studies have demonstrated that the insulin-like growth factor mRNA-binding protein IMP3 can promote the proliferation of human leukemia cells. As part of a program to develop clinical biomarkers to predict metastasis and prognosis of renal-cell carcinomas, Jiang et al. have investigated whether expression levels of IMP3 in renal-cell carcinomas are related to tumor metastasis.

By using immunohistochemical analysis, the investigators quantified levels of IMP3 in 406 primary renal carcinomas obtained from radical or partial nephrectomy and 95 samples of metastatic renal-cell carcinoma. A total of 371 patients with localized primary tumors were also followed up over a median of 63 months to determine whether their cancer had spread. Compared with nonmetastatic renal-cell tumors, markedly elevated levels of IMP3 expression were found in metastatic tumors, as well as in a subset of primary tumors that were likely to subsequently develop metastases. Approximately $80 \%$ of patients with primary tumors that tested positive for IMP3 developed metastatic cancer, compared with $13 \%$ of patients with IMP3-negative tumors. After adjustment for well-known clinical variables, statistical analysis showed that over 5 years patients with IMP3-positive tumors were almost six times more likely to develop metastasis and four times more likely to die than those with IMP3-negative tumors.

In summary, these data indicate that IMP3 has a role in the metastasis of renal-cell carcinoma and could prove to be a useful prognostic marker, with therapeutic implications for the selection of patients to undergo early systemic treatment following a nephrectomy.

Original article Jiang Z et al. (2006) Analysis of RNA-binding protein IMP3 to predict metastasis and prognosis of renal-cell carcinoma: a retrospective study. Lancet Oncol 7:556-564

\section{Formalin instillation to treat hemorrhagic radiation proctopathy}

Radiation proctopathy is a common complication of radiotherapy for pelvic malignancies, such as prostate cancer, and is associated with symptoms including diarrhea, urgency, rectal pain, and rectal bleeding. Several treatment options for hemorrhagic radiation proctopathy have been investigated (e.g. 5-aminosalicyclic acids, argon-beam laser treatment, and argon-plasma coagulation) but all have associated risks. The use of formalin instillation for hemorrhagic radiation proctopathy has also been reported, mostly using large volumes $(250-2,000 \mathrm{ml})$ or a long contact time with the rectal mucosa; these techniques have been associated with significant complication rates.

Cullen et al. carried out a retrospective pilot study to investigate whether the instillation of small-volume, dilute formalin $(20 \mathrm{ml}$ of a $5 \%$ formalin solution), could control bleeding in 20 patients with hemorrhagic proctopathy. The solution was instilled as a bolus via the operating channel of a flexible sigmoidoscope; the sigmoidoscope was reinserted after approximately 3 min mucosal contact time, to remove the formalin. 